\title{
Re: Phase II Trial of Neoadjuvant Gemcitabine and Cisplatin in Patients with Resectable Bladder Carcinoma
}

\author{
Daniel Herchenhorn, Rodrigo Dienstmann, Fabio A. Peixoto, Franz S. de Campos, Valdelice O. Santos, \\ Denise M. Moreira, Hedilene Cardoso, Isabele A. Small, Carlos G. Ferreira
}

Departments of Clinical Oncology (DH, FAP), Clinical Cancer Research (RD, VOS, IAS, CGF), Urology (FSC) and Radiology (DMM), National Cancer Institute, and Eli Lilly Medical Support (HC), Rio de Janeiro, RJ, Brazil

Int Braz J Urol, 33: 630-638, 2007

To the Editor:

Malignancy is not aware of the artificial boundaries between surgery and oncology. The malignant tumor lives and expands by its own rules and biological possibilities. To approach any malignancy with curative intent, one must be aware of these facts. A multidisciplinary approach reaching beyond the borders of pride and enthusiasm over ones own capacity as a surgeon or oncologist is the proper way to improve treatment options for the patient. Because, at the end of the day, an increase in survival benefit is what the patient is actually seeking for and mainly nothing else.

Neoadjuvant chemotherapy in muscle invasive urothelial urinary bladder carcinoma adds a survival benefit for our patients as we can see in a number of trials published and presented in recent years. The ABC-group in the meta analysis from 2003 showed that clearly with a combined hazard ratio of 0.87 in favor of the neoadjuvant regime and an absolute benefit of $5 \%$ at 5 years, improving survival from $45 \%$ to $50 \%$ (1). In the second report from the very same group 2005, in which the SWOG-trial (2) also was included, the power of the trial increased and the hazard ratio of 0.86 was even more favorable. The absolute benefit of $5 \%$ at 5 years remained (3).

The two Nordic trials Nordic Cystectomy Trial 1 and 2 constituted a large part of the mentioned
ABC meta analysis. Separately the Nordic trials were also merged into a meta analysis of their own, published in 2004 (4). The outcome in some aspects was different compared to the larger ABC-trial, mainly following; the ARR (absolute risk reduction) was 8 $\%$ in the trial as a whole and the subgroup analysis showed a distinct advantage in the T3-subgroup (UICC, 1982) with an ARR of $11 \%$. The hazard ratio for the whole trial was 0.80 in favor of neoadjuvant chemotherapy and for the T3-subgroup 0.69. Fiveyear overall survival for patients with clinical T3 in the experimental arm was $48 \%$ and in the control arm $37 \%$. For the T2-subgroup the hazard ratio was 0.85 (but without statistical significance).

The explanation for this outcome comparing to the larger ABC trials was as follows; "The studies are comparatively large and clinically homogenous since they were done within the same recruitment areas, within a similar biological domain and cystectomy was baseline treatment in both studies."

The routine use of neodjuvant chemotherapy (platinum-based combination chemotherapy) in urothelial urinary bladder carcinoma is now standard treatment of T2b - T3b -tumors in two major Swedish university hospitals, Karolinska University Hospital and Uppsala University Hospital. Still one needs to address novel approaches that are emerging. One is the use of new and more efficient chemotherapy 
regimes and the other is the extent of lymph node dissection.

In the present trial of Herchenhorn et al., gemcitabine was combined with cisplatin, which is a relatively new constellation. By utilizing gemcitabine, the tolerability increases and enables patients of higher age to be treated in future trials and treatment regimes. One of the major caveats in above-mentioned trials was the relatively low age of the study populations, which also has been commented on previously (5). Still in the present trial (Herchenhorn et al.), the ages were ranging from 18-70 with a median age of 63 and it would be of interest to also follow a population of higher age. The question of extent of lymphadenectomy is still debated, although some prestigious investigators have utilized their non-randomized retrospective single centre experiences to advocate this regimen emphatically. When it comes to staging it is for sure the best tool we have for establishing nodal status and nodal extent of the present malignancy. Still we find ourselves in a biological dilemma. On one hand we have patients with macrometastatic dissemination to a number of lymph nodes heralding a generalized disease. Certainly a generalized disease cannot be treated by local surgical resection. On the other hand we have patients with micrometastatic disease and some proponents of extended dissection dearly wish that surgical skills would remove that very disease. Investigations into the immunobiology of nodal dissemination in urothelial urinary bladder cancer has in the same time shown the existence of a strong defense mechanism directed against the assaulting tumor dissemination (6). Thus there is a slight risk that an overzealous removal of nodal deposits can lead to the surgeon depriving the patient of an existent immunological response! Randomized trials entailing the use of neaodjuvant cisplatin combination therapy carries so far the only conclusive evidence for improving the survival chances in our patients. It is of that reason of great interest to follow new attempts, like the present trial, to improve the neoadjuvant regimen in terms of tolerability and lower toxicity.

\section{REFERENCES}

1. Advanced Bladder Cancer Meta-analysis Collaboration: Neoadjuvant chemotherapy in invasive bladder cancer: a systematic review and meta-analysis. Lancet. $2003 ; 361$ : 1927-34.

2. Grossman HB, Natale RB, Tangen CM, Speights VO, Vogelzang NJ, Trump DL, et al: Neoadjuvant chemotherapy plus cystectomy compared with cystectomy alone for locally advanced bladder cancer. N Engl J Med. 2003; 349: 859-66.

3. Advanced Bladder Cancer Meta-analysis Collaboration: Neoadjuvant chemotherapy in invasive bladder cancer: update of a systematic review and meta-analysis of individual patient data advanced bladder cancer (ABC) meta-analysis collaboration. Eur Urol. 2005; 48: 202-5; discussion 205-6.

4. Sherif A, Holmberg L, Rintala E, Mestad O, Nilsson J, Nilsson S, Malmstrom PU; Nordic Urothelial Cancer Group: Neoadjuvant cisplatinum based combination chemotherapy in patients with invasive bladder cancer: a combined analysis of two Nordic studies. Eur Urol. 2004; 45: 297-303.

5. Droz JP: Editorial comment to Advanced Bladder Cancer Meta-analysis Collaboration. Neoadjuvant chemotherapy in invasive bladder cancer: update of a systematic review and meta-analysis of individual patient data advanced bladder cancer (ABC) meta-analysis collaboration. Eur Urol. 2005; 48: 205-6.

6. Marits P, Karlsson M, Sherif A, Garske U, Thörn M, Winqvist O: Detection of immune responses against urinary bladder cancer in sentinel lymph nodes. Eur Urol. 2006; 49: 59-70.

Dr. Amir Sherif

Karolinska University Hospital Department of Urology 17176 Stockholm, Sweden

E-mail: amir.sherif@swipnet.se 